\title{
Statistical Characterization of the Antenna and Wireless Multipath Virtual Channel Interaction
}

\author{
Eddy Luis Molina Morales ${ }^{(1)}$, Leandro de Haro Ariet*(1) Alejandro Milanés Arce ${ }^{(1)}$ \\ (1) Madrid University of Technology \\ \{eddy,alejandro,leandro\}@gr.ssr.upm.es
}

\begin{abstract}
Introduction
The characterization and modeling of radio channels with multipath [1], was a hi importance. So, the study of the radio channel performance of the channel allows for a better designs of the radio networks. For these reasons, measurement of the performance that antennas in the radio channel (both in the mobile station, as in the base station) when operating in conditions of multipath is very important [2]. We propose a reverberation procedure digitally created to simulate the fading caused for the interaction of the antenna and the radio channel. It is done through a file actual containing antenna pattern measurements.
\end{abstract}

\section{System of Reverberation}

The procedure to create the digital form of the reverberation consists of a 360 by 180 points matrix, representing all the possible spatial directions from where the radio waves could arrive and get into the antenna system. The procedure is based on iterations that simulate the time evolution. The waves arriving to the antenna are generated in random way. All of them have random amplitudes, random phases, random spreading and random arrival directions in order to simulate a propagation environment with intense multipath (Fig. 1). In the next iteration, a different channel is generated simulating the time and space varying channel. The procedure operate with two virtual parallel channels, one to simulate the main polarization components and the other for the cross polarization component, both of them, randomly [3].

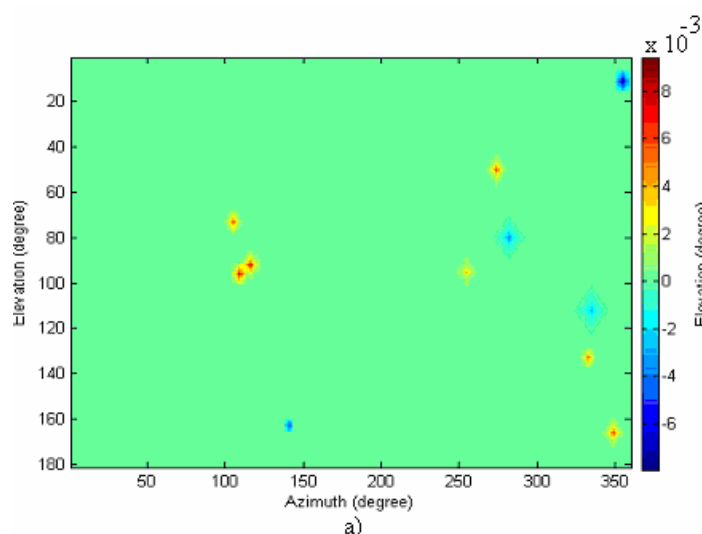

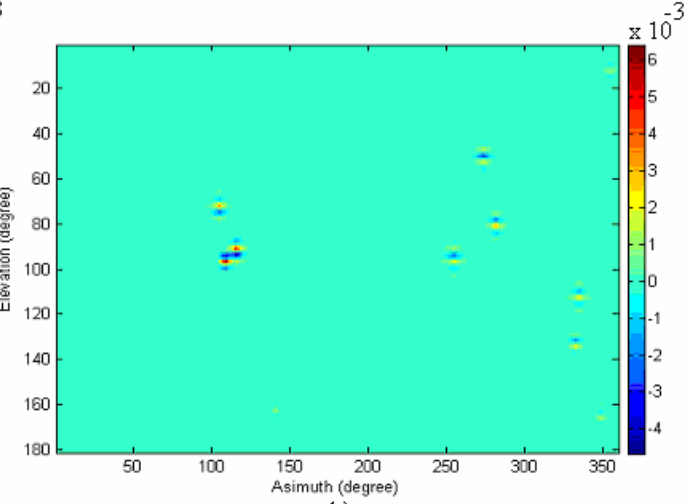

b)

Figure 1. 2-D Representation of virtual channel with several waves arriving, a) Copolarization to the antenna. b) Cross-polarization to the antenna. (The signal amplitude are in $\mathrm{V} / \mathrm{m}$ ) 


\section{Antenna Characteristics}

The canonical antenna used to obtain and characterize the channel fading is de half-wave dipole. The corresponding radiation pattern shape is shown in Fig. 2. Only the normalized directional characteristics and the phase response in the main polarization and cross polarization planes are used [3]. These dipole patterns are these which that interact with the virtual radio channel.
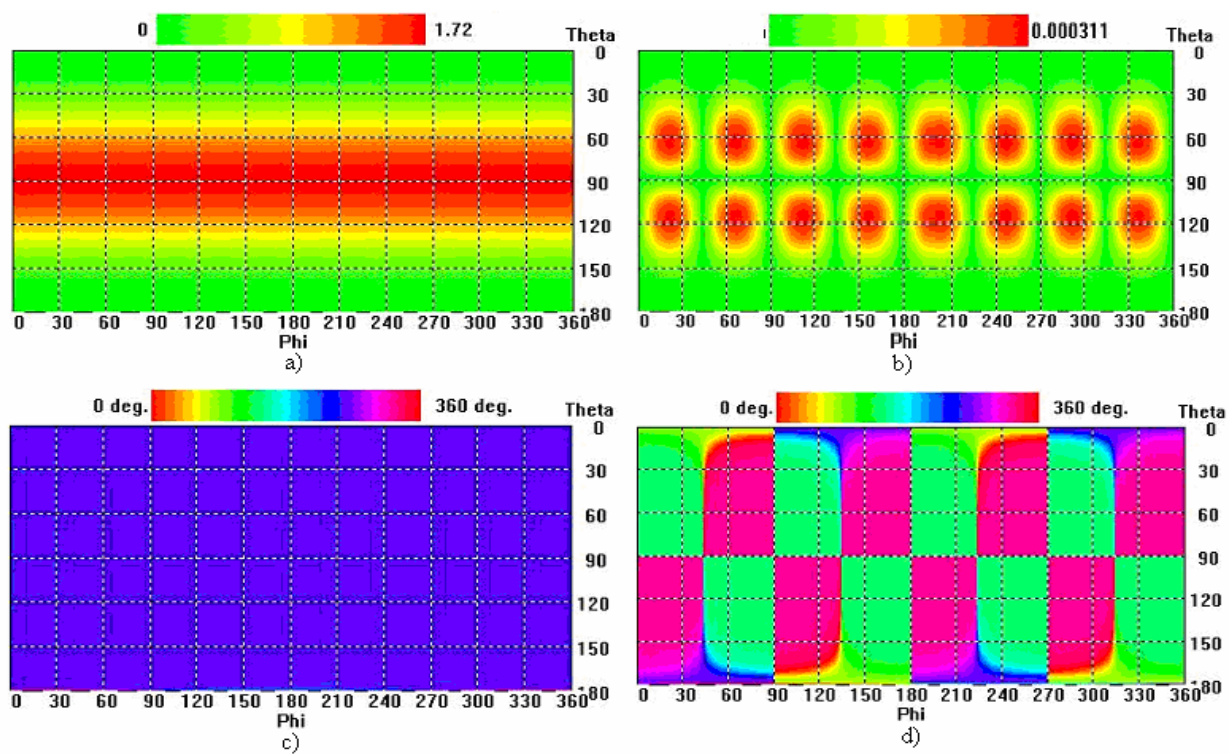

Figure 2. Electrical field radiation pattern of the half wave dipole. a) Amplitude directional characteristics with main polarization. b) Amplitude directional characteristics with cross- polarization. c) Phase directional characteristics with main polarization. d) Phase directional characteristics with cross- polarization.

It cant be seen from Fig. 2 that the antenna radiation pattern are directional both in amplitude and phase [4]. Therefore, it turns out that the signal with fading, seen at the antenna terminals, should depend strongly on those directional characteristics.

\section{Signals at the Antenna Terminals}

Let $H(\theta, \varphi, t)$ be a function describing all the space-time distribution characteristics of amplitude, phase and polarization of the signals pseudo-randomly generated by the virtual radio channel. $F(\theta, \varphi)$ is the normalized amplitude, polarization and phase response directional characteristics of the antenna [4]. Therefore, the signal amplitude at antenna terminals can be described by the following expression [5]:

$$
s(t)=\xi \int_{0}^{2 \pi \pi} \int_{0}^{\pi} H(\theta, \varphi, t) \cdot F(\theta, \varphi) \cdot \operatorname{sen} \theta d \theta d \varphi
$$

where $\xi$ is a constant having implicitly the antenna maximum gain and input impedance, and the signal maximum amplitude. The implemented algorithm works with a file containing measurements of normalized amplitude, polarization and phase directional characteristics of the antenna and with a dynamic function describing the instantaneous 
radio channel. A graphical description of the interaction geometry between channel and the antenna is shown in Fig. 3.
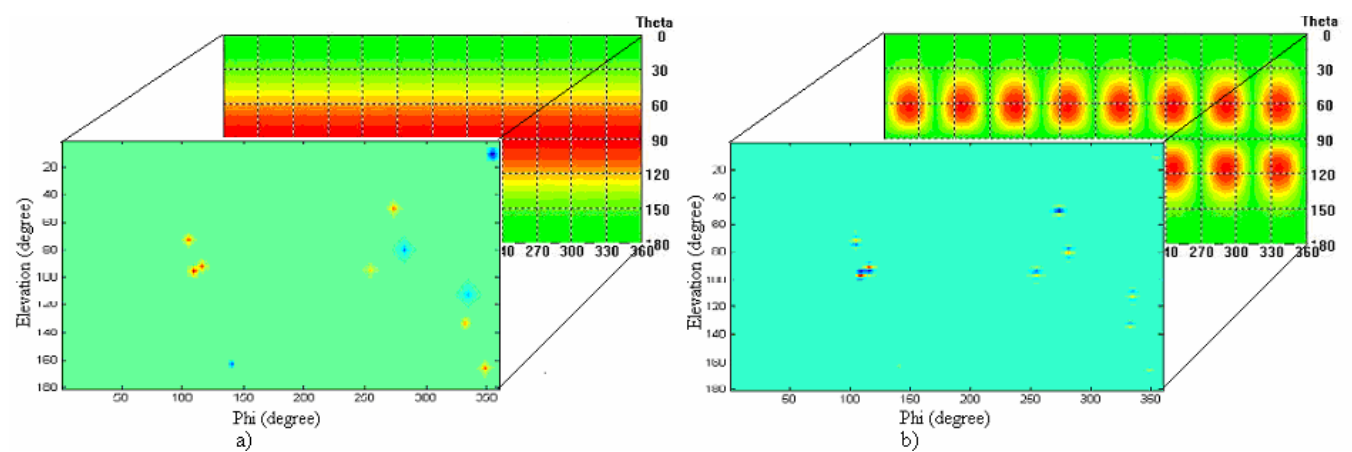

Figure 3. Simulated channel-antenna interaction geometry. a) Main polarization components. b) Cross-polarization components.

As a result, the fading signals is shown in Figs. 4 were obtained. Notice that the amplitude and depth of fading are very deep, very close to what is obtained when a real channel plenty of scatter elements is measured.
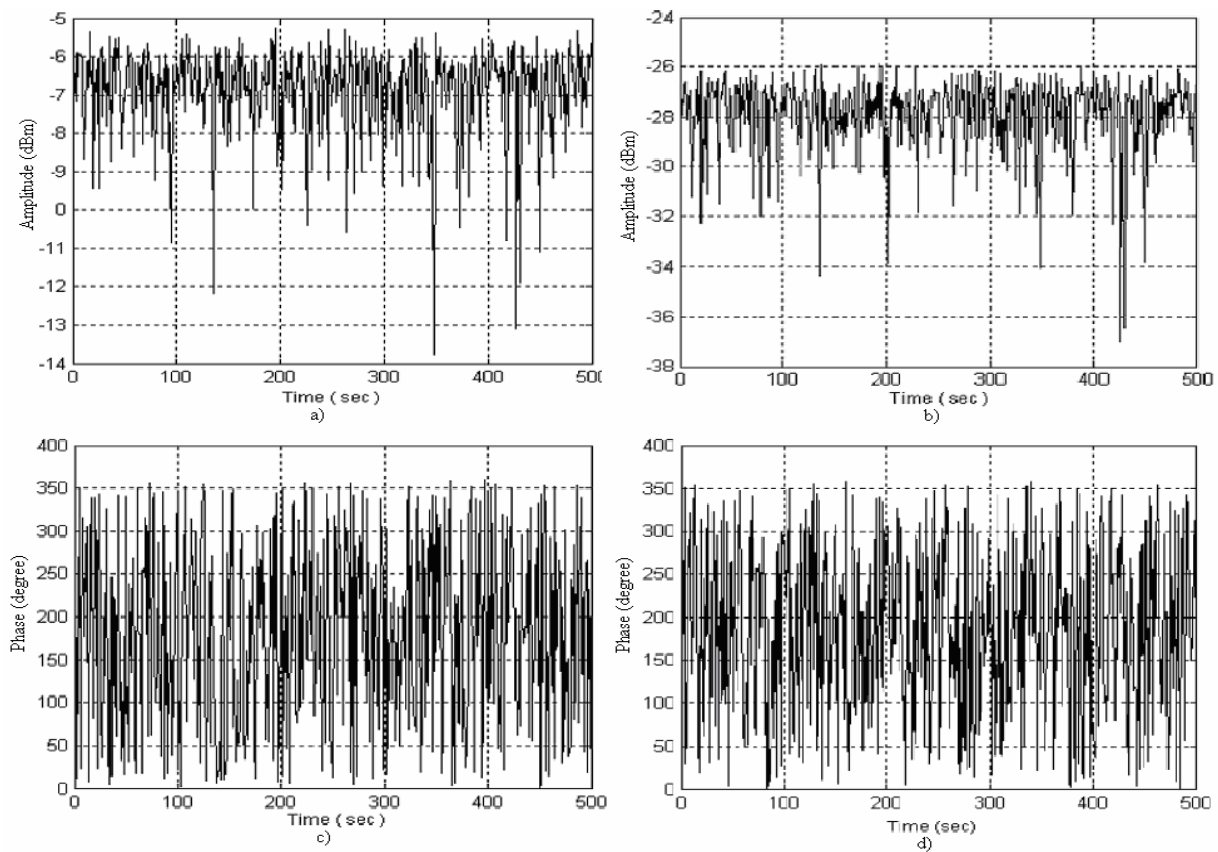

Figure 4. Signal at dipole output terminal. a) Amplitude main polarization componentes. b) Amplitude cross-polarization componentes. c) Phase main polarization componentes. d) Phase cross-polarization componentes.

\section{Statistical Characterization}

After the adjustment of the statistical distributions of amplitudes and phases of the signals measured at the port of the used antenna, it can be seen from Fig 5 a) and c), that the cumulative distributions functions (cdf) of the amplitude are the $m$-Nakagami kind. Because it gets the best fit of the collected samples. From Figure $5 \mathrm{~b}$ ) and d) the statistical distribution that gets the best fits the cdf of the phase for both types of polarization is the 
uniform distribution. The obtained result are useful because realistic radio channels have a similar behavior in the lack of a dominant path.
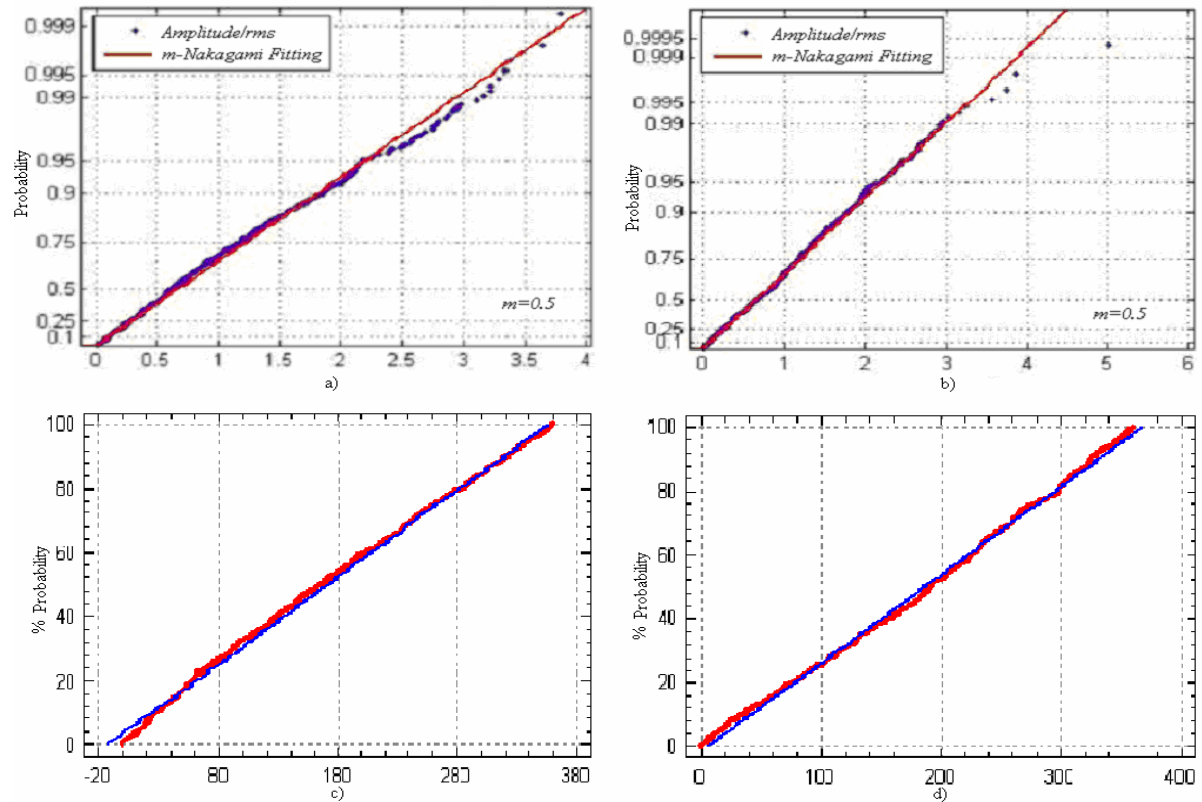

Figure 5. Statistical distribution fitting. a) m-Nakagami b) m-Nakagami c) Uniform d) Uniform.

\section{Conclusions}

The proposed procedure allows us to remark easily the interaction between the antenna and the simulated channel. The procedure not only permits to simulate the interaction between ideal antennas and channels but it could make to interact real antennas with the simulated channel by using a file with measured pattern. Moreover, it is applicable to evaluate radiation systems orientated to MIMO (Multiple Input and Multiple Output), because it allows the calculation of the spatial correlation of the different types of antennas with virtual ratio channels. In the case of real antennas, in the lack of the file of the measurements of their directional characteristics, the same results can be obtained from approximately through electromagnetic simulation. Then you can use the program MatLab to obtain the behavior of a real antenna in a dispersive environment.

\section{References:}

[1] Blaunstein, N., Radio Propagation in Cellular Networks, Norwood, MA:Artech House, 1999

[2] Vaughan, R., and J. B. Andersen, Channels, Propagation, and Antennas for Movil Communications, London: IEE, 2002.

[3] A. C. Ludwig, "The definition of cross polarization". IEEE Transactions on antennas and propagation, AP-21, 116-119, January 1973.

[4] E. P. Stincer, “Antenas, Teoría General” Edit. Imprenta ITM (La Habana). 2001. pp. 124-127.

[5] Paul D, Thus Shara D. "Spatial Correlation for General Distributions of Scatterers,'IEEE Signal Processing. vol. 9, NO. 10, pp.305-308, Oct.2002. 